\title{
La definición de "hombre" y la estructura del Liber differentiarum II de Isidoro de Sevilla
}

\section{(1) Sergey Vorontsov \\ St.Tikhon's Orthodox University, Rusia \\ ORCID: 0000-0002-6035-325X}

Recibido el 29 de noviembre de 2019; aceptado el 24 de mayo de 2020.

\begin{abstract}
Resumen
El presente artículo versa sobre la estructura del tratado isidoriano llamado Liber differentiarum II (Diff. II). Hasta el momento, los trabajos acerca del contenido de la obra y su distribución han explicado su estructura según consideraciones que podríamos llamar "externas", en tanto se basan en ideas generales en relación con el género o la lógica del texto. En contraste con ello, el objetivo de este estudio es ofrecer una explicación "interna" de tal estructura, una fundada en su contenido conceptual. Nuestra hipótesis sostiene, pues, que la base para articular el texto entero es la definición de "hombre" que Isidoro desarrolla en Diff. II.13, así como su lugar en la escala de seres (Diff. II.16). En efecto, la posición especial del ser humano, que es considerado tanto por Isidoro como por sus fuentes como una creatura universal intermedia entre Dios (espíritu intelectual puro) y los seres materiales carentes de razón, es de capital importancia para la comprensión y consecuente estructuración de la obra.
\end{abstract}

PALABRAS CLAVE: ISIDORO DE SEVILLA, AGUSTÍN, ANTIGÜEDAD TARDÍA, DIFFERENTIAE, ESCALA DE SERES.

\section{The Definition of "man" and the Structure of the Liber differentiarum II of Isidore of Seville}

\begin{abstract}
This article deals with the structure of the Isidorian treatise called Liberdifferentiarum II (Diff. II). Up to now, researches about the content of the work and its distribution have explained its structure according to considerations that it could be called "external", as they are based on general ideas around text genre or logic. However, I aim to offer an "internal" explanation of such a structure based on its conceptual content. The hypothesis holds, then, that the basis for articulating the entire work is the definition of "man" that Isidore develops in Diff. II.13, as well as its place on the chain of beings (Diff. II.16). Indeed, the special position of the human being, who is
\end{abstract}


considered both by Isidore and by his sources as a universal creature intermediate between God (pure intellectual spirit) and material beings lacking reason, is of capital importance for understand and structure the work.

KEYWORDS: ISIDORE OF SEVILLE, AUGUSTINE, DIFFERENTIAE, LATE ANTIQUITY, CHAIN OF BEING.

\section{Introducción}

Isidoro de Sevilla (c. 560 - 636) tiene una justificada reputación de gran enciclopedista. Pero, además, se debe notar que en su obra enciclopédica suele utilizar el método retórico-gramatical de etimología (cf. Sánchez Martínez, 2000: 224-275). En otras palabras, el pensamiento Isidoriano se caracteriza tanto por el enciclopedismo y, por supuesto, por el modo derivativo de sus ideas, como por su exposición gramatical (Fontaine, 2000: 167-182).

La composición de sus dos libros Differentiae-de los cuales nos interesa particularmente el segundo- oscila entre una y otra característica. ${ }^{1}$ Así, mientras que el primero pertenece a un género gramatical que trata sobre varios casos de homonimia y sinonimia (cf. Codoñer, 1985), ${ }^{2}$ el segundo se aleja del principio gramatical de la construcción de la differentia tan nítido en el anterior (Codoñer, 1992a: 20).

En realidad, la variedad de los temas tratados allí corresponde más bien a un principio "enciclopédico". Jacques Fontaine opina que en Diff. II Isidoro representa el mundo como "una pirámide gigante de diferencias" (1983: 772). Sin embargo, eso no explica la estructura del libro ni el conjunto de los temas, dado que el género enciclopédico es algo muy vago y amplio (Codoñer, 2011: 115-116). A ello se le añade la dificultad de que la obra consta capítulos independientes y por lo tanto carece a menudo de una transición clara entre uno y otro. ¿Cómo es posible que en una obra tan breve estén ligados la doctrina de la Trinidad y la división de la filosofía, el esquema de la jerarquía de seres y el problema de la relación entre predestinación y libre albedrío? ¿Es que acaso hay algún hilo conductor que ordene problemáticas tan disímiles? Esta cuestión termina de complicarse ante la ausencia de un proemio particular que pudiera aclarar de alguna forma la intención del Diff. II.

Lamentablemente, no se puede decir que en los estudios isidorianos se preste mucha atención este texto, tal como lo señala María Adelaida Andrés Sanz en la introducción a la edición crítica (2006a: 9*). Tal vez ello se explique por el hecho de que los dos libros de las Differentiae se consideren, por lo general, como una unidad completa. Es como si el libro segundo careciera de una intención propia. Pero no es así. En lo que sigue, voy a presentar una hipótesis sobre el hilo conductor del tratado llamado Diff. II de Isidoro de Sevilla.

\section{Estado de la cuestión}

De una forma u otra, en la historiografía se expusieron tan solo algunas consideraciones sobre la estructura y la intención del Diff. II. Giorgio Brugnoli supuso que este segundo libro representa un breviario filosófico en el que se examinan sucesivamente Teología (capítulos 1-12), Filosofía Natural (capítulos 13-19), Filosofía

\footnotetext{
1 Aunque la datación de dichos tratados es problemática, por lo general son considerados obras tempranas. Cf. Andrés Sanz, 2006a: $22^{*}-26 \%$.

2 Carmen Codoñer sostiene que este género aparece recién a partir del siglo IV. Cf. Codoñer, 1986. Véase también Magallón García, 1996: 235-260.
} 
del Conocimiento (capítulos 20-24), Psicología (capítulos 25-28), Filosofía Moral (capítulos 29-41) (1964: 76). El problema principal de tal reconstrucción consiste en que no explica el conjunto de temas dentro de un mismo apartado. Por ejemplo, ¿por qué la doctrina del Universo está ausente en el apartado de la Filosofía Natural mientras que sí está presente, por ejemplo, en el libro De natura rerum, lo que prueba que el problema del Universo y su estructura no escapó de la atención de Isidoro? ¿Por qué la división general de la Filosofía, de la Física y de la Lógica están tratadas en el marco de la Filosofía Moral? Además, la propia estructura reconstruida apenas se corresponde con la división de la Filosofía en la época de la Antigüedad Tardía.

Una propuesta más constructiva para determinar la estructura del Diff. II fue realizada por C. Codoñer, quien supuso que en esta obra se describe la visión general del mundo. A partir de esto, la investigadora estableció una analogía con los libros VII-XI de las Etimologías y señaló su afinidad con el plano del comentario a los primeros capítulos del libro de Génesis (1992a: 25). ${ }^{3}$ Eso explica la presencia de los capítulos dedicados a la creación y la jerarquía de seres. Sin embargo, ¿por qué el libro se termina con los capítulos dedicados a la Filosofía y sus ramas? Contestar esta pregunta usando el modelo explicativo formulado por Codoñer no es posible sin tener en cuenta distintos factores.

Finalmente Andrés Sanz, quien realizó la edición crítica del Diff. II, señala la secuencia Dios - hombre - conocimiento, la cual, como indica la investigadora, corresponde a los modelos culturales de los siglos VI-VII. Sin embargo, según su propia observación, eso aún no explica por qué la secuencia es exactamente así (2006a: 52*).

En mi opinión, el mismo texto contiene ciertas claves para explicar tal unión de elementos heterogéneos en una obra y su secuencia determinada. Con todo, antes de comenzar con la formulación de la solución que propongo, conviene tener presente la estructura del libro que se encuentra en la edición crítica de Andrés Sanz:

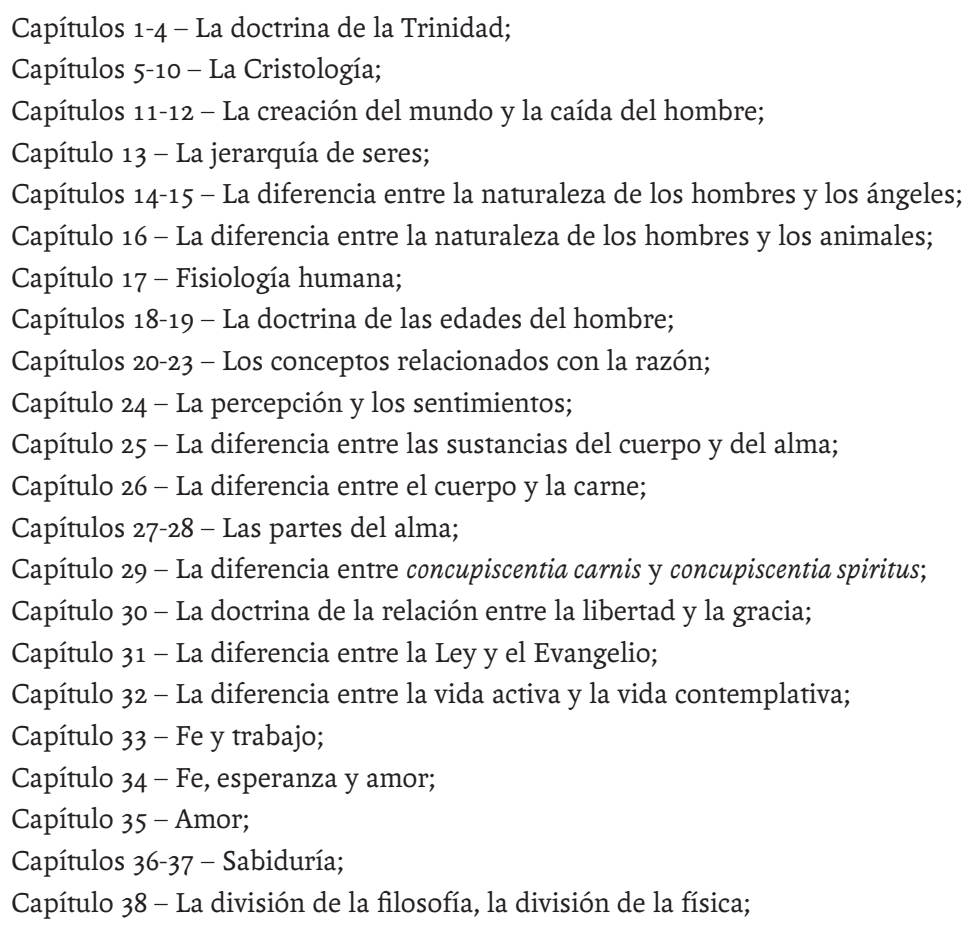


Debemos advertir, no obstante, que apoyarnos en el conjunto y el orden de los capítulos de la edición crítica -que establece un orden de los capítulos del texto que deriva la tradición manuscrita- (cf. Andrés Sanz, 2006a: 187*) para la reconstrucción de la estructura de la obra es algo arriesgado, pues puede condicionar a priori cualquier posible estructuración alternativa. En efecto, piénsese que las differentiae relativamente cortas y poco relacionadas entre sí en la edición manuscrita pueden "perderse", aparecer o reordenarse como el resultado del trabajo de edición. ${ }^{4}$ En las tablas de estructuración del manuscrito y de las ediciones originales que presenta Andrés Sanz se puede observar la numeración, el orden y la existencia de algunas partes del texto (2006a: $\left.314^{*}-323^{*}\right) .5$ Pero aunque en la mayoría de los casos el orden de aparición del material manuscrito coincide con el resultado final, la numeración de los capítulos y los párrafos varía.

No todos los manuscritos incorporan el extenso capítulo 17, donde se describe fisiología humana. ${ }^{6}$ De todos modos, aun cuando la ausencia de dicho capítulo reduce considerablemente el tema del cuerpo humano, no se puede afirmar que con eso el tema de la dualidad del hombre desaparezca del todo del Diff. II.

Otro inconveniente está en el hecho de que algunos manuscritos contienen una diferencia complementaria, la de uir/homo después del capítulo 16 (que en la edición de Andrés Sanz está indicado como "17*") y un grupo de capítulos sobre los temas éticos del Diff. I al final del libro. ${ }^{7}$ Estos complementos no rompen con la estructura existente, sino que, por el contrario, forman parte de ella.

\section{La clave antropológica}

Hechas estas precisiones, podemos pasar ya al análisis del texto. Si prestamos atención al contenido conceptual, no es difícil observar que la parte dedicada a Dios (capítulos 1-10) es considerablemente menos extensa que la parte dedicada al hombre (capítulos 11-35). Además, estos capítulos tienen diferente extensión. Por ejemplo, los capítulos cristológicos son relativamente cortos (el más largo contiene 39 líneas), mientras que entre los antropológicos se encuentran algunos mucho más amplios, como el 17 (146 líneas) y el 28 (76 líneas). Pero si solo dijéramos que Diff. II está dedicado principalmente a la antropología, ${ }^{8}$ faltaría aún explicar su estructura y su intención. Veamos.

Entre los capítulos dedicados al hombre hay tres que resultan esenciales. Son aquellos en los cuales se lo define: capítulo 13, sobre la determinación de su lugar en la jerarquía de seres; capítulo 14, sobre la diferencia entre la naturaleza de los hombres y la de los ángeles; y capítulo 16, sobre la diferencia entre el hombre y el animal.

\footnotetext{
4 Los mejores ejemplos de estos procesos en el legado de Isidoro pueden ser De uiris illustribus y el De differentiis I. Véase Codoñer, 1963: 20-40; y 1992b: 77-82. Sin embargo, este problema persiste incluso para las Etymologiae. Véase, por ejemplo, Codoñer, 1994: 125-146. El De differentiis II, a su vez, supo ser reestructurado en forma de preguntas-respuestas. Véase: Andrés Sanz, 2006b.

5 Véase también Andrés Sanz, 2000.

6 Se trata de tres manuscritos de los siglos VIII y IX. Véase Andrés Sanz, 2006a: 167*-177*.

7 Se trata de tres manuscritos del s. IX. Véase Andrés Sanz, 2006a: $177^{*}-182^{*}$.

8 Sobre la antropología de Isidoro en su obra enciclopédica véase Gasti, 1998.
} 
En la jerarquía de seres el problema del lugar del hombre ocupa la posición central, como muestra este pasaje clave:?

Gradus uel differentiae rerum sex sunt, id est, non uiuentia, uiuentia, inrationabilia, mortalia, inmortalia; nouissimus qui est super omnia Deus. Primus gradus est eorum quae non crescunt, uitaque motuque carent, qualis est in lapidibus. Secundus est in his quae crescunt, uitamque motumque sine sensu habent, quails est in herbis uel arboribus, quibus si minime uita insensibilis motusque inesset, nec germinare utique nec crescere potuissent; at ista quae uitam sine sensu habent lapidibus praeponuntur et terrae. Tertius gradus est in his quae non solum crescunt uel uiuunt sed etiam sentiunt, qualis est in pecoribus. Quartus gradus est in his quae crescunt, uiuunt, sentiunt, uel intellegunt, sed tamen mortales, ut animalia, sunt, qualis est in hominibus. Quintus est in his quae sentiunt, et intellegunt et inmortalia sunt, qualis est in angelis. Sextus id quod incommutabile et infinitum et simplex est, quo omnis haec natura inspiratur, gubernatur et regitur, quod est Deus (Diff. II.13.36-40: 26.1-27.16).

Los grados o diferencias de las cosas son seis, esto es: no viviente, viviente, irracionales, mortales, inmortales, y finalmente lo que es sobre todas las cosas, esto es, Dios. El primer grado está <compuesto> de aquellas cosas que no crecen y que carecen de vida y movimiento, tales como las piedras. El segundo está en esas cosas que crecen, y tienen vida y movimiento y no sienten, tales como las hierbas o los árboles, a los que si les correspondiera mínimamente vida y movimiento insensible no podrían germinar ni, sobre todo, crecer; con todo, estas cosas que tienen vida y no sienten se prefieren a las piedas o la tierra. El tercer grado está en esas cosas que no solo crecen y viven sino también sienten, tales como el ganado. El cuarto grado está en esas cosas que crecen, viven, sienten y entienden, pero sin embargo son mortales, como los animales, tales como son los hombres. El quinto es en esas que sienten, y entienden y son inmortales, tales como los ángeles. El sexto es aquello que es inconmutable, infinito y simple, aquel por quien es animada, gobernada y regida toda esta naturaleza, que es Dios (Diff. II.13.36-40). ${ }^{10}$

Ahora bien, para construir esta differentia Isidoro combina varias fuentes. Ella se basa en el esquema de Gregorio Magno que distingue los niveles del siguiente modo: existe, pero no vive (las piedras), vive, pero no siente (los árboles), siente (animales irracionales), comprende y diferencia (los hombres y los ángeles). ${ }^{11} \mathrm{~A}$ ello Isidoro añade el nivel de Dios (superomnia) basándose en los esquemas de Agustín (De civ. Dei VII.23; VIII.6) y posiblemente en el de Claudiano Mamerto (De statu animae, Epilogus). El motivo de la búsqueda de Dios, importante para ambos autores, es prácticamente ignorando por Isidoro.

Nuestro autor solo se limita a indicar que Dios dirige la naturaleza de todas las creaturas (natura inspiratur, gubernaturet regitur). Pero mientras que Cicerón atribuye la dupla gubernare et regire a la providencia, los autores cristianos se la imputaron a Dios. ${ }^{12}$ Por su parte, los verbos inspirare et gubernare en relación con la naturaleza se

9 Sobre la jerarquía de seres, véase la obra clásica de Lovejoy, 1933. Para las implicaciones relevantes a la Antigüedad Tardía, véase O'Meara, 1987.

10 Traducción del autor.

11 Gregorio, Moralia in Iob VI.16.20: 492.12-22: “Omne namque quod est, aut est et non uiuit; aut est et uiuit, sed nequaquam sentit; aut est et uiuit et sentit, sed non intellegit nec discernit; aut est et uiuit, sentit et intellegit, et discernit. Sunt namque lapides nec tamen uiuunt. Sunt arbusta uiuunt quidem nec tamen sentiunt. Herbarum namque atque arborum uita uiriditas uocatur, sicut per paulum de seminibus dicitur: insipiens, tu quod seminas, non uiuificatur nisi prius moriatur. Sunt bruta animalia, uiuunt et sentiunt nec tamen intellegunt. Sunt angeli et uiuunt et sentiunt et intellegendo discernunt. Homo itaque. Quia habet esse cum lapidibus, uiuere cum arboribus, sentire cum animalibus, discernere cum angelis, recte nomine uniuersitatis exprimitur in quo iuxta aliquid ipsa uniuersitas tenetur".

12 Sirvan como ejemplo de este contraste los siguientes pasajes: Cicerón, De natura deorum II.73: 78.72-74: "prouidentiam fingi quasi quandam deam singularem, quae mundum omnem gubernet et regat"; Agustín, De civ. Dei V.21: 158.32-33: "haec plane deus unus et uerus regit et gubernat, ut placet...". 
encuentran en el Octavio de Minucio Félix. Allí Octavio sostiene que la diferencia entre los animales y el hombre consiste en la posibilidad de conocimiento del numen que inspira y gobierna toda la naturaleza a través de la belleza de la creatura. ${ }^{13}$ Dado que los contextos de esta frase en el Octavio y en Diff. II coinciden parcialmente, podemos admitir la posibilidad de que Isidoro estuviera aludiendo a este texto.

Isidoro complementa de manera considerable las cualidades de los niveles de lo irracional - lo racional - lo inmortal, a los cuales corresponden las bestias, el hombre y los ángeles respectivamente, mientras que no ofrece ninguna característica positiva de los non uiuentia. La mayor cantidad de atributos -cinco en total-aparece en la esfera de lo racional, o sea, el hombre. Lo irracional y lo inmortal, en cambio, solo tienen tres atributos cada uno. Dejando a un lado la diferencia entre los hombres y los ángeles que había sugerido Agustín, Isidoro utiliza la traducción de la Isagoge hecha por Boecio para estructurar los atributos humanos, es decir, "crescunt", "uiuunt", "sentiunt", "intelligunt"y "sunt mortales". Tal esquema es el siguiente: se alimentan y preservan la vida (árboles), tienen sentimientos (animales), comprenden (hombres), no se alimentan pero tienen todo lo demás (ángeles), y para la característica de dioses se vale de la jerarquía de Porfirio, en tanto "differentia [...] rationalis uero et inmortalis dei" (Boecio, Porph. Isagoge, 17). Es evidente, pues, que el nivel de lo racional ha sido elaborado de una manera más minuciosa tanto en lo que se refiere a las fuentes utilizadas como en el grado de detalle.

La posición del hombre en la jerarquía de seres, determinada en el capítulo 13, condiciona la diferencia entre la naturaleza humana y la de las bestias, por una parte, y la diferencia entre la humana y la angélica, por la otra (capítulos 14-15). Al diferenciar entre la naturaleza humana y la angélica, Isidoro tiene un objetivo más bien retórico: mostrar a sus lectores las grandes posibilidades de naturaleza humana y, a la vez, la miseria de la voluntad del ser humano.

Por cierto, los hombres por un lado son "ratione capaces, intellectu sapientes" (Isidoro, Diff. II.14.43: 30.19), pero por otro son "Homines [...] natura magis quam doctrina ualentes [...] ad studia inertes [...] tardi ad sapientiam" (Diff. II.14.43: 30.19-26). Con todo, las características intrínsecas del hombre como un ser vivo se enumeran en el capítulo 16: a. dualidad (está compuesto de carne y de alma); $b$. racionalidad; $c$. libre albedrío; $d$. capacidad de vicio y de virtud, (característica esta última que puede proceder de tratado de Claudiano Mamerto donde el contexto es también la jerarquía de los seres). ${ }^{14}$ Lo importante de señalar es que la estructura de esta caracterización corresponde en cierto modo a la estructura de todo el libro. Y ello, de la siguiente manera:

a. Los capítulos 17-19 tratan del cuerpo humano, su composición, propósito y evolución, es decir, de las edades. En el capítulo 25 se explica la diferencia entre la sustancia del cuerpo y del alma; el capítulo siguiente ("corpus / anima") representa un comentario al capítulo anterior. La descripción de la estructura del alma se hace en los capítulos 27-28, los cuales reflejan, por lo tanto, lo expuesto en el capítulo 17. Así se revela el primer atributo humano, mencionado en el capítulo 16: la naturaleza compuesta del hombre que tiene el cuerpo y el alma.

13 Minucio Felix, Oct.17.2-4: 13.23-36: "praecipue cum a feris beluis hoc differamus, quod illa prona in terramque uergentia nihil nata sint prospicere nisi pabulum, nos, quibus uultus erectus, quibus suspectus in caelum, <quibus > datus est sermo et ratio, per quae deum adgnoscimus, sentimus, imitamur, ignorare nec fas nec licet ingerentem sese oculis et sensibus nostris caelestem claritatem [...] Quid enim potest esse tam apertum, tam confessum tamque perspicuum, cum oculos in caelum sustuleris et quae sunt infra circa que lustraueris, quam esse aliquod numen praestantissimae mentis, quo omnis natura inspiretur, moueatur, alatur, gubernetur?".

14 Isidoro, Diff. II.16.46: 32.1-33.4: "Homo est animal ex corpore animaque uiuenti conpositum atque spirituali conparatione formatum, subsistens ratione liberique arbitrii uoluntate, uitiorum capax atque uirtutum". Compárese con Mamerto, De statu animae. Epilogus: 194.15-195.5: "spiritus pecoris, qui non subsistit post corpus, praetermittendus ibi est, ut ad rationalem spiritum ueniamus [...] accidunt animo disciplinae, accidit iustitia et iniustitia, castitas et libido [...] conligitur enim subiectum semper esse animum aut uitiis aut uirtutibus" 
b. Los capítulos 20-24 tratan sobre la razón y los sentimientos (en el capítulo 24 incluso ambos están puestos a la misma altura) y, por consiguiente, pueden estar incluidos en el grupo de los capítulos psicológicos. Pero, por otro lado, estos capítulos revelan la racionalidad del hombre. En el tratado agustiniano De ordine, que fue una de las fuentes principales del capítulo 20 ("rationale / rationabile"), la racionalidad se considera la característica distintiva del ser humano con respecto a los animales en el contexto de la discusión sobre la posición del hombre entre el mundo animal y el mundo divino. ${ }^{15}$ Más aún, Agustín deja en claro que esta posición es dinámica: el hombre puede ascender a Dios o descender al estado animal (cf. Kuntz, 1987: 45). Por lo tanto, es manifiesto que este grupo de capítulos se relaciona con el atributo de la racionalidad de la definición del hombre en el capítulo 16.

d. Por su parte, el capítulo 29, en el cual se diferencian la concupiscencia carnal y la concupiscencia espiritual, ${ }^{16}$ termina el grupo de los capítulos psicológicos y al mismo tiempo introduce varias differentiae teológicas. Este capítulo, que se sitúa en el contexto moral, se basa en la contraposición esencial entre el alma y el cuerpo. La concupiscencia espiritual se interpreta como una intención positiva a diferencia de la lujuria, la concupiscencia carnal, que es negativa. Isidoro opone la expresión "motus animi"-que representa concupiscentia carnis-a "intentio mentis" -que se refiere a concupiscentia spiritus-. ${ }^{17}$ Aquí "mente" puede aludir (una vez más) a la racionalidad del hombre, mientras que la conexión entre animus y caro parece tener connotaciones negativas.

Los capítulos teológicos 32-35 están vinculados por el tema de la ética, y se centran en las cuestiones de la relación entre la vida activa y la contemplativa, entre la fe y el trabajo, y la doctrina de lo que más tarde llegó a conocerse como virtudes teologales (aunque Isidoro en este caso no les aplica el término virtus). A este grupo de capítulos, en mi opinión, se lo puede llamar teológico-ético. Por lo tanto, en estas differentiae se revela el aspecto moral de la definición del hombre (uitiorum capax atque uirtutum).

c. El libre albedrío, una de las características esenciales del hombre, se analiza en el capítulo 30, que trata sobre la relación entre este y la gracia. En este capítulo, además, se desarrolla la doctrina de la predestinación. El capítulo 31, dedicado a la comparación de conceptos de la Ley (o sea, la Ley de Moisés) y del Evangelio, se vincula con el anterior mediante la noción clave de la gracia que, por su parte, se encuentra relacionada con el Evangelio.

Ahora bien, el tema central para los capítulos 36-41, que clausuran Diff. II, es el de la sabiduría. Al mismo tiempo, la definición de "sabiduría" se revela tanto en la perspectiva de la oposición clásica entre lo divino y lo humano (la sabiduría y el conocimiento, capítulo 36$),{ }^{18}$ como en el contexto de la relación entre sabiduría y elocuencia

15 Agustín, De ordine II.11.31: 124.18-125.30: "Hic genere posito, quod animal dictum est, uidemus additas duas differentias, quibus credo admonendus erat homo, et quo sibi redeundum esset et unde fugiendum. Nam ut progressus animae usque ad mortalia lapsus est, ita regressus esse in rationem debet; uno uerbo a bestiis, quod rationale, alio a diuinis separatur, quod mortale dicitur. Illud igitur nisi tenuerit, bestia erit, hinc nisi se auerterit, diuina non erit. Sed quoniam solent doctissimi uiri, quid inter rationale ac rationabile intersit, acute subtiliter que discernere, nullo modo est ad id quod instituimus neglegendum. Nam rationale esse dixerunt, quod ratione uteretur uel uti posset, rationabile autem, quod ratione factum esset aut dictum".

16 Esta differentia se remonta a Gál. 5: 17: "caro enim concupiscit aduersus spiritum spiritus autem aduersus carnem haec enim inuicem aduersantur ut non quaecumque uultis illa faciatis". La concupiscentia es uno de los conceptos morales básicos de la patrística latina. Véase, por ejemplo, Nisula, 2012.

17 Isidoro, Diff. II.29.109: 69.1-4: "concupiscentia carnis est motus animae turpis in affectum sordidae delectationis; concupiscentia uero spiritus est ardens intentio mentis in desideria sanctae uirtutis".

18 Cf. Cicerón, De officiis I.153. 
(capítulo 37). En el primer caso, la sabiduría está vinculada a la contemplación, ${ }^{19}$ en el segundo, se equipará con la Filosofía. ${ }^{20}$ Más adelante, Isidoro sigue una división de la Filosofía en tres partes: física, lógica y ética, donde se exponen los componentes de cada una de estas partes. En el caso de la ética, son las virtudes cardinales (mejor dicho, lo que se conciben como tales), ${ }^{21}$ lo que permite desarrollar el tema de los vicios en el capítulo final del libro.

Parece que este grupo de capítulos se aleja un poco del modelo ofrecido, puesto que carece de las características correspondientes a la definición de hombre en el capítulo 16, aun cuando el contenido de esos capítulos tiene el mismo carácter estoico; de hecho, la sabiduría, en su definición clásica, se concibe como la realización del componente tanto moral como racional del ser humano. Isidoro, por lo visto, se percataba de ello. Por un lado, eso se manifiesta a nivel de las relaciones intertextuales. Así, la definición de "dialéctica" como parte de la lógica en el capítulo 39, y la definición de "razón" (ratio) en el capítulo 20 coinciden parcialmente. ${ }^{22}$ Por otro lado, Isidoro presta especial atención a la definición de ética y sus secciones (capítulos 40-41). Allí observa como partes de la ética las cuatro virtudes en contraposición con los cuatro vicios estoicos. ${ }^{23} \mathrm{~A}$ ese conjunto Isidoro prefiere (al menos en este texto) los ocho vicios derivados de la tradición monacal. ${ }^{24}$ De este modo, se pueden relacionar la cualidades del hombre en el capítulo 16 y la temática de los capítulos 17-41 de la siguiente manera: conjunto del cuerpo y el alma, capítulos 17-19, 25-28; raciocinio, capítulos 20-24; libre albedrío, capítulo 30 (y capítulo 31 como complemento); capacidad de vicio y virtud, capítulos 29,32-35; sabiduría (relacionada tanto con la prudencia como con la moralidad), capítulos 36-41.

Lo dicho hasta aquí, entonces, permite determinar la conexión del capítulo 16 con los capítulos posteriores. No obstante, queda por explicar de qué forma se relacionan con él los capítulos anteriores.

La definición de hombre en este capítulo comienza con la afirmación "homo est animal", el hombre es un ser vivo. Cualquier creatura está de alguna forma integrada en la jerarquía de seres, concretamente el lugar del hombre como ser vivo dentro de ella se revela en los capítulos 13-15 y, en parte, en el 16, ya que allí el hombre se equipara con un animal.

Por su parte, la jerarquía de seres definida por Isidoro también explica, según parece, la presencia en el texto exposiciones sobre Dios, la creación y la caída del hombre. Dios se posiciona en el escalón más alto de esta jerarquía, Él creó a todos los demás seres y los dirige. De esta manera, la presencia de los capítulos 11 ("creatio / formatio") y 12 ("differentia paradisorum", donde se relata, entre otras cosas, la caída del hombre) resulta ser el antecedente del lugar que el hombre ocupa dentro de la jerarquía de seres y, por decirlo así, de su actual condición de miseria.

La exposición sobre la fuente de toda la creación y el escalón más alto en la jerarquía de seres -Dios- se sitúa en la parte inicial (capítulos 1-4). Le sigue la exposición sobre

\footnotetext{
19 Isidoro, Diff. II.36.147: 95.4-5: "sapientia autem tantummodo aeterna contemplatur". Cf. Agustin, De Trinitate XII.

20 Isidoro, Diff. II.37.149: 96.7-8: "Porro sapientiam ueteres philosophiam uocauerunt”.

21 Isidoro repite la misma fórmula en las Etymologiae II.24.5. Véase Bejczy, 2011: 28-67.

22 Isidoro, Diff. II.21.86: 55.2-3: "Ratio uero est motus quidam animi uisum mentis acuens, ueraque a falsis distinguens". Diff. II.39.153: 99.3-100.4: "Dialectica est ratio siue regula disputandi, intellectum mentis acuens, ueraque a falsis distinguens".

23 Isidoro, Diff. II.41.159: 104.2-4: "Contra haec tamen quattuor uirtutum genera totidem uitia philosophi opposita dicunt: metum scilicet et gaudium et cupiditatem siue dolorem".

24 Isidoro, Diff. II.41.161: 106.21-24: “Septem autem sunt perfecta uel principalia uitia, ex quibus copiosa uitiorum turba exoritur, id est gulae concupiscentia, fornicatio, auaritia, inuidia, ira, tristitia, inanis gloria, nouissima dux ipsa et earum regina superbia". Véase al respecto la obra clásica de Zöckler, 1893; y también la compilación más reciente de Newhauser, 2005.
} 
Jesucristo (capítulos 5-10), no solo en forma de complemento útil y actual relacionado con la reciente conversión de los visigodos del arrianismo al cristianismo niceno, ${ }^{25}$ sino también a la luz del estudio sobre el hombre. En efecto, la problemática de Jesucristo se construye alrededor de dos grandes temas: 1. el de la relación entre naturaleza divina y humana en los capítulos 5-7, a partid de los términos "unigenitus" y "primogenitus", es decir, alrededor de la cuestión tradicional sobre la relación de los pasajes de las Sagradas Escrituras donde Jesucristo es igual al Padre y donde es menos que Él (en este caso Jn. 1: 14, y Rom. 8: 29). Allí Isidoro diferencia entre los nombres de Jesucristo según su humanidad y su divinidad. También se concentra en la diferencia de la naturaleza humana de Jesucristo, quien no fue tocado por el pecado original; y 2. el tópico del hombre caído en los capítulos 8-10, donde diferencia entre el nacimiento, la muerte y la resurrección de Jesucristo y "nosotros".

En resumen, la estructura de los capítulos iniciales se compone de la siguiente manera: la naturaleza divina (del creador y de la creatura, ubicada en el escalón más alto de la jerarquía de seres), capítulos 1-4; Jesucristo como hombre-Dios, capítulos 5-10; la creación del mundo y la caída del hombre, capítulos 11-12; el mundo presentado en forma de la jerarquía espiritual y el lugar en ella del hombre (como ser vivo), capítulos 13-16.

Ello muestra, entonces, que la definición de hombre del capítulo 16 articula, por decirlo así, la estructura de todo el libro Diff. II.

\section{Conclusiones}

La hipótesis según la cual el plan del tratado se articula alrededor de la definición de hombre en el capítulo 16 permite: 1) recomponer una determinada estructura del libro, 2) señalar el tema que une capítulos a primera vista muy poco sistemáticos, y 3) determinar hasta cierto punto cuál es la idea que recorre todo el Diff. II.

$\mathrm{Al}$ exponer esta idea hay que decir que la posición especial del hombre en la escala de seres presente en autores anteriores fue bien recibida por Isidoro. ${ }^{26}$ En sus Sententiae explicará el papel del hombre en el proceso de adoración a Dios por parte de los seres, y explicará también que aquellos que no tienen su propia razón adoran a Dios a través del hombre. ${ }^{27}$ Las fuentes del Diff. II desarrollarán, además, otros aspectos de esta tesis. Gregorio Magno, en su exposición de la jerarquía de seres, subraya el carácter universal del hombre (cf. nota11). Por su parte, Agustín, en De ordine, se refiere a la similitud del hombre con Dios según su racionalidad, y con los animales según su mortalidad (cf. nota 15).

Se puede afirmar, pues, que el Diff. II tiene como uno de sus objetivos principales desarrollar en detalle la definición de hombre, tal como este es presentado en los capítulos 13-16, contemplando su lugar en la jerarquía de seres, su fisiología y psicología, la relación de la sustancia del cuerpo con la del alma, el problema de la libre voluntad y el destino, y finalmente los asuntos éticos (es decir, el modo de vivir, y la relación

25 La conversión de los visigodos al cristianismo niceno tuvo lugar en el Tercer Concilio de Toledo en el año 589, apenas menos de una década antes de que Isidoro comenzara la redacción del Diff. II, elaborado entre 598 y 615 . Sobre el Concilio, véase, por ejemplo la edición conmemorativa del centenario del Concilio, Arzobispado de Toledo, 1989.

26 Formigari, 1973: 325: "In the nature of man different grades of being meet: as microcosm, he recapitulates the continuity of orders inherent in the scale of nature".

27 Isidoro, Sententiae I.4.3: 13.17-24: “Quibusdam gradibus intellegentiae per creaturam progreditur homo ad intellegendum Deum Creatorem, id est ab insensibilibus surgens ad sensibilia, a sensibilibus surgens ad rationabilia, a rationabilibus surgens ad Creatorem. Intellegibilia per se conlaudant Deum; inrationabilia et insensibilia non per se, sed per nos, dum ea conside rantes Deum laudamus; sed ideo dicuntur laudare ipsa, quia eandem laudem earum parturit causa". Isidoro utiliza el texto de Agustín, Enarr. in psalmos 148.3. 
entre la fe y los asuntos necesarios para la salvación de la virtud). Posteriormente, el orden de los capítulos de la mayor parte de libro (capítulos 17-35) sigue más o menos la enumeración de las características en la definición de hombre en el capítulo 16.

El carácter dinámico del hombre que oscila entre lo divino y lo animal puede explicar por qué Isidoro presta tanta atención a los temas triadológicos y cristológicos, así como a los de la creación del mundo y del hombre, y al de su caída. Así Isidoro marca las ambas relaciones del hombre. Además, en la estructuración de la obra parece ser lógico comenzar hablando de Dios, el creador y el rector del mundo, y no de las creaturas, aun cuando una de ella sea el hilo conductor del texto.

Isidoro deja para el final el desarrollo del concepto de sabiduría (y, por supuesto, el de filosofía), tema que también se vincula con el hombre: por un lado, se relaciona con sus dos cualidades esenciales, el raciocinio y la moralidad; por otro, alude otra vez al dinamismo del ser humano en la jerarquía de seres, ya que la filosofía fue entendida, precisamente, como un arte de ascender hacia lo divino.

Así se puede ver que Isidoro en Diff. II partiendo del principio léxico y gramatical de differentia y de la amplitud "enciclopédica" de los temas tratados, termina por transcenderlos a ambos al utilizar como hilo conductor de su obra un principio filosófico como lo es el antropológico. 


\section{Bibliografía}

\section{Fuentes}

"Augustinus Hipponensis (1955). De ciuitate Dei. Eds. Dombart, B. y Kalbe, A. Turnhout: Brepols. (CCSL 48).

" Augustinus Hipponensis (1956). Ennarationes in psalmos. Eds. Dekkers, E. y Fraipont, J. Turnhout: Brepols. (CCSL 40).

» Augustinus Hipponensis (1968). De Trinitate. Ed. Mountain, W. J. Turnhout: Brepols. (CCSL 50A).

"Augustinus Hipponensis (1970). Contra academicos. De beata vita. De ordine. De magistro. De libero arbitrio. Eds. Green, W. M y Daur, K. D. Turnhout: Brepols. (CCSL 29).

» Boethius (1966). Porphyrii Isagoge. Ed. Minio-Paluello, L. Brujas-París: Desclée de Brouwer.

» Cicero (1933). De natura deorum. Ed. Ax, W. Leipzig: Teubner.

" Cicero (1963). De officiis. Ed. Atzert, E. Leipzig: Teubner.

》Claudianus Mamertus (1885). De statu animae. Epistula ad Sapaudum. Ed. Engelbrecht, A. Wein: Tempsky. (CSEL 11).

»Gregorius Magnus (1992). Commento morale a Giobbe (vol.1). Ed. bilingüe SiniscalCo, P., intr. Dagens, C., trad. Gandolfo, E. Roma: Città Nuova Editrice.

»Isidorus Hispalensis (1998). Sententiae. Ed. Cazier, P. Turnhout: Brepols. (CCSL 111).

»Isidorus Hispalensis (2006). Liber Differentiarum [II]. Ed. Andrés Sanz, M. A. Turnhout: Brepols. (CCSL 111A).

》 Minucius Felix (1982). Octauius. Ed. Kytzler, B. Leipzig: Teubner.

\section{Bibliografía complementaria}

"Andrés Sanz, M. A. (2000). "Relación y transmisión manuscrita de los tres libros de Differentiae editados en P.L. 83 (Isidoro de Sevilla)", Revue d'Histoire des Textes 30, 239-262.

"Andrés Sanz, M. A. (2006a). "Introducción general. Bibliografía. Apéndice" y "Conspectus siglorum". En: Isidorus Hispalensis. Liber Differentiarum [II]. Ed. Idem. Turnhout: Brepols. (CCSL 111A), 7*-323* y 3-5.

»Andrés Sanz, M. A. (2006b). “Una reelaboración de textos isidorianos en forma de 'interrogationes et responsiones'", Helmantica 72, 29-48.

"Bejczy, I. (2011). The Cardinal Virtues in the Middle Ages. A Study in Moral Thought from the Fourth to the Fourteenth Century. Leiden-Boston: Brill. (Brill's Studies in Intellectual History 202).

" Brugnoli, G. (1964). "Il Liber de differentiis rerum di Isidoro di Siviglia", Vetera Christianorum 1, 65-82. 
" Codoñer, C. (1963). El De uiris illustribus de Isidoro de Sevilla. Salamanca: CSICInstituto «Antonio de Nebrija».

" Codoñer, C. (1985). "Les plus anciennes compilations de Differentiae: formation et évolution d'un genre littéraire gramatical", Revue de Philologie, de Littérature et d'Histoire Anciennes 59, 201-219.

»Codoñer, C. (1986). "La conception de la Differentia dans le recueil Inter Aptvm d'Isidore de Séville", Revue de Philologie, de Littérature et d'Histoire Anciennes 60, 187-196.

»Codoñer, C. (1992a). “Differentia y etymologia, dos modos de aproximación a la realidad". En: Holtz, L. y Fredouille, J.-C. (eds.). De Tertullien aux mozarabes. Mélanges offerts à Jacques Fontaine à l'occasion de son 70e anniversaire (vol.1). París: Institut d'études augustiniennes; Turnhout: Brepols. (EAA 132), 19-30.

»Codoñer, C. (1992b). "Introducción”. En: Isidoro de Sevilla. Diferencias. Libro I. Ed. Idem. París: Les Belles Lettres, 77-82.

» Codoñer, C. (1994). "Fases en la edición de las Etymologiae, con especial referencia al libro X", Evphrosyne: Revista de filología clásica 22, 125-146.

"Codoñer, C. (2011). “La enciclopedia. Un género sin definición. Siglos I a.C.-VII d.C.”. En: Fossati, C. (ed.). Giornate filologiche genovesi: l'enciclopedismo dall'Antichità al Rinascimento. Génova: Dipartimento di Antichità, Filosofia e Storia, 115-154.

»Concilio-Arzobispado de Toledo (ed.) (1989). Concilio III de Toledo: XIV Centenario, 589-1989. Toledo: Caja Toledo.

"Fontaine, J. (1983). Isidore de Séville et la culture classique dans l'Espagne wisigothique. París: Institut d'Etudes Augustiniennes; Turnhout: Brepols. (EAA 100-101).

"Fontaine, J. (2000). Isidore de Séville. Genese et originalité de la culture hispanique au temps des Wisigoths.Turnhout: Brepols. (TH 8).

" Formigari, L. (1973). "Chain of Being". En: Wiener, P. P. (ed.). Dictionary of the History of Ideas. Studies of Selected Pivotal Ideas (vol. 1). Nueva York: Charles Scribner's Sons, 325-335.

» Gasti, F. (1998). L'Antropoligia di Isidoro. Le fonti del libro XI delle Etimologie. Como: Edizioni New Press.

» Kuntz, P. G. (1987). "'From the Angel to the Worm'. Augustine's Hierarchical Vision". En: Kuntz, M. L. e Idem (eds.). Jacob's Ladder and the Tree of Life. Concepts of Hierarchy and the Great Chain of Being. Nueva York - Bern - Frankfurt am Main: Peter Lang, 41-53.

" Lovejoy, A. O. (1933). The Great Chain of Being. A Study of the History of an Idea. Cambridge, Mass.: Harvard University Press.

" Magallón García, A.-I. (1996). La tradición gramatical de differentia yetymologia hasta Isidoro de Sevilla. Zaragoza: Departamento de ciencias de la Antigüedad - UniZar.

"Newhauser, R. (ed.) (2005). In the garden of evil. The vices and culture in the Middle Ages.Toronto: Pontifical Institute of Mediaeval Studies.

» Nisula, T. (2012). Augustine and the Functions of Concupiscence. Leiden - Boston: Brill.

" O'Meara, D. J. (1987). "The Chain of Being in the Light of Recent Work on Neoplatonic Hierarchies". En: Kuntz, M. L. y Kuntz, P. G. (eds.). Jacob's Ladder and the Tree of Life. Concepts of Hierarchy and the Great Chain of Being. Nueva York - Bern - Frankfurt am Main: Peter Lang, 15-30. 
»Sánchez Martínez, C. (2000). La etimología latina. Concepto y métodos. Murcia: Servicio de Publicaciones de la Universidad de Murcia.

»Zöckler, O. (1893). Das Lehrstück von den sieben Hauptsünden: Beiträge zur Dogmen- und zur Sittengeschichte, in besonders der vorreformatorischen Zeit. Múnich: Becker. 
